\title{
ANALYSIS IN COMPLEX BANACH SPACES
}

\author{
ANGUS E. TAYLOR
}

1. Introduction. Abstract spaces, and Banach spaces in particular, have played a prominent rôle in recent years in connection with many problems of analysis. There has also been a notable tendency for the concepts and tools of analysis to take a place alongside the algebraic and topological notions which are characteristic of the whole subject of abstract spaces. Thus analysis becomes more algebraic, and at the same time its range is broadened.

For a great many purposes it is immaterial whether a Banach space be real or complex. It is well known that a large portion of the theory of linear operations, as developed in Banach's book (Banach [1] $]^{1}$, is equally valid for complex or real spaces. There are, however, situations in which the complex number system plays a crucial rôle. The theory of analytic functions in Banach spaces is a case in point. There are two large divisions of this subject: the theory of functions of a complex variable, the values of the functions lying in a Banach space, and the theory of analytic functions of an abstract variable. Our principal concern in this paper will be the first of these two theories. A brief survey of the second theory, and references to the literature, are given in $\$ 8$.

Henceforth, except as otherwise stated, we shall use the term Banach space to mean a complex Banach space. The algebraic structure of such a space is that of an additive Abelian group with the complex numbers as operators. The topology of the space is defined by a norm; the norm of an element $x$ is written $\|x\|$. It has the properties of an absolute value. Then $\|x-y\|$ is the distance between $x$ and $y$, and the space is assumed to be a complete metric space.

2. Analytic functions. The basic development of the theory of analytic functions with values in a complex Banach space $E$, the independent variable being a complex number, follows the pattern of classical analysis. It seems to have been pointed out first by Wiener [1] that Cauchy's integral theorem is valid in this general setting. The usual consequences, such as the Cauchy integral formula, Liouville's theorem, and the Taylor and Laurent expansions, then follow. It is only when we come to theorems that deal in some way with di-

An address delivered before the Stanford meeting of the Society on April 24, 1943, by invitation of the Program Committee; received by the editors May 18, 1943.

1 Numbers in brackets refer to the bibliography at the end of this paper. 
vision of function values that significant differences appear.

3. Weak, uniform, and strong analyticity. If $E$ is an abstract Banach space, the space of all complex linear functionals defined on $E$ is also a Banach space, denoted by $E^{*}$. Let us consider a single-valued function $x(z)$ with values in $E$, where $z$ ranges over a region $D$ of the complex plane. If $f$ is an element of $E^{*}, f x(z)$ is a numerical function of $z$. If $f x(z)$ is regular in $D$ for each $f$ in $E^{*}$ we shall say that $x(z)$ is weakly regular in $D$.

THEOREM 1. The function $x(z)$ is regular in $D$ if and only if it is weakly regular there.

This theorem, in a slightly more general form, is due to Nelson Dunford (Dunford [1, Theorem 76]). Instead of requiring $f x(z)$ to be regular for each $f$ in $E^{*}$, it is sufficient to impose the requirement for each $f$ in a determining manifold in $E^{*}$. A subset $S$ of $E^{*}$ is called a determining manifold if it is a closed linear subspace of $E^{*}$, and if there is a constant $M$ such that for each $x$ in $E$ the least upper bound of $|f(x)| /\|f\|$, as $f$ ranges over $S$, is not less than $M\|x\|$.

The theorem depends upon the following lemma.

LEMMA 1. If $T$ is an arbitrary range, and $x(t)$ is a function on $T$ into $E$ such that $f x(t)$ is bounded on $T$ for each $f$ in $E^{*}$ (or, more generally, for each $f$ in a determining manifold), then $\|x(t)\|$ is bounded on $T$.

The proof of the lemma (Dunford [1, p. 354]) is of the category type. We shall give a new, brief proof of Theorem 1. Let us write

$$
Q(z, h)=(x(z+h)-x(z)) / h .
$$

Suppose that $x(z)$ is weakly regular in $D$. Let $C$ be a circle of radius $r$, with center at $z$, which, together with its interior, lies in $D$. By Cauchy's integral formula

$$
f Q(z, h)-f Q(z, k)=\frac{1}{2 \pi i} \int_{C} f x(t) \frac{(h-k) d t}{(t-z-h)(t-z-k)(t-z)},
$$

provided that $|h|$ and $|k|$ are less than $r$. Now, for each $f, f x(t)$ is bounded on $C$; hence, by the lemma, there is a constant $M$ such that $\|x(t)\| \leqq M$ on $C$. Thus $|f x(t)| \leqq M\|f\|$, and

$$
|f[Q(z, h)-Q(z, k)]| \leqq \frac{M|h-k|}{(r-|h|)(r-|k|)}\|f\| .
$$

But then 


$$
\|Q(z, h)-Q(z, k)\| \leqq \frac{M|h-k|}{(r-|h|)(r-|k|)} .
$$

It follows, since $E$ is complete, that $Q(z, h)$ approaches a limit as $h \rightarrow 0$. This proves that $x(z)$ is regular in $D$. That weak regularity is a consequence of ordinary (strong) regularity is, of course, trivial.

The next theorem, discovered by the writer (Taylor [ 6 , Theorem $2.1]$ ), is closely related to Theorem 1 . Let $E_{1}$ and $E_{2}$ be Banach spaces, and let $\left[E_{1}, E_{2}\right]$ denote the Banach space of all linear transformations of $E_{1}$ into $E_{2}$. The norm $\|T\|$ of a transformation $T$ is defined as the least upper bound of $\|T x\|$ for all elements $x$ of $E_{1}$ such that $\|x\|=1$. Let $A_{\lambda}$ be a family of linear transformations of $E_{1}$ into $E_{2}$, the parameter $\lambda$ ranging over an open set $D$ of the complex plane. We say that $A_{\lambda}$ is strongly analytic and regular in $D$ if for each $x$ in $E_{1}$ the function $A_{\lambda} x$ is regular as a function with values in $E_{2}$. We say that $A_{\lambda}$ is uniformly analytic and regular in $D$ if it is regular as a function with values in $\left[E_{1}, E_{2}\right]$.

THEOREM 2. The family $A_{\lambda}$ is uniformly regular in $D$ if and only if it is strongly regular there.

The proof of this theorem may be modeled very closely after the above proof of Theorem 1. Instead of Lemma 1 we use an analogous lemma about linear transformations (cf. Banach $[1$, p. 80 , Theorem 5]). It is a corollary of Theorems 1 and 2 that the regularity of $\phi A_{\lambda} x$ for each $x$ in $E_{1}$ and each $\phi$ in $E_{2}^{*}$ implies the strong, and hence the uniform, regularity of $A_{\lambda}$. It is to be emphasized that Theorems 1 and 2 are not true if we substitute continuity for analyticity.

4. Concrete Banach spaces. A space $E$ is said to possess a denumerable basis $\left\{x_{n}\right\}$ if to each $x$ in $E$ there corresponds a unique sequence of numbers $\left\{u_{n}\right\}$ such that

$$
x=\sum_{k=1}^{\infty} u_{k} x_{k} .
$$

If we define $f_{n}(x)=u_{n}, f_{n}$ is an element of $E^{*}$ (Banach [1, p. 111]). A number of familiar spaces have bases. with the property that a series $\sum_{k=1}^{\infty} a_{k} x_{k}$ converges if the norms of the partial sums $\sum_{k=1}^{n} a_{k} x_{k}$ are bounded (Dunford and Morse [1, p. 415]). A space with a basis of this sort will be said to have property $(A)$. The function spaces $L^{p}(p>1)$, and the sequence spaces $l^{p}(p \geqq 1)$ are examples. The space $(c)$ of convergent sequences has a basis, but it does not have property $(A)$. 
TheOREM 3. Let $E$ be a space with a denumerable basis. Let $\left\{u_{n}(z)\right\}$ be a sequence of numerical functions of the complex variable $z$, each analytic and regular in a region $D$, and such that

(a) to each compact ${ }^{2}$ subset $S$ of $D$ and each $f$ in $E^{*}$ there corresponds a constant $M$ such that $\left|f\left(\sum_{k=1}^{n} u_{k}(z) x_{k}\right)\right| \leqq M$ if $z$ is in $S$ and $n=1,2$, $3, \ldots$;

(b) the series $\sum_{k=1}^{\infty} u_{k}(z) x_{k}$ converges if $z$ is in $D$.

Then the function $x(z)=\sum_{k=1}^{\infty} u_{k}(z) x_{k}$ is analytic and regular in $D$. Its derivative is $x^{\prime}(z)=\sum_{k=1}^{\infty} u_{k}^{\prime}(z) x_{k}$.

We first observe that if the space has property $(A)$, condition (b) is automatically satisfied when (a) is, by virtue of Lemma 1 . Because of Theorem 1, it is enough to prove that $x(z)$ is weakly regular. This, however, follows directly from (a) and (b), by Montel's theorem. We leave it for the reader to show that $x^{\prime}(z)$ is given by the series indicated.

Theorem 3 furnishes a practical method for testing analyticity. For example, it shows at once that a sequence $\left\{u_{n}(z)\right\}$ of functions with a common region of regularity $D$ may be regarded as an analytic function on $D$ into $l^{p}$ if the partial sums $\sum_{k=1}^{n}\left|u_{k}(z)\right|^{p}$ are uniformly bounded in each compact subset of $D$. For other results of the same type, see Taylor [6, Theorem 3.1].

Consider next the space $C$ whose elements are complex functions $x(t)$ continuous on the closed interval $[a, b]$, with the norm

$$
\|x\|=\max _{a \leqq t \leqq b}|x(t)| .
$$

If $x(t, z)$ is a function of $t$ and $z$ which belongs to $C$ for each value of $z$, we shall use the notation $x(\cdot, z)$ when we wish to regard $x(t, z)$ as a function of $z$ with values in $C$. Bôcher [1] used the term semianalytic to describe functions $x(t, z)$ which are continuous in the pair $t, z$ and analytic as functions of $z$. Bôcher's results concerning such functions, insofar as the dependence upon $z$ is concerned, may be regarded as corollaries of the abstract theory with which we are concerned, as soon as it is shown that the hypothesis of semi-analyticity is sufficient to make $x(\cdot, z)$ an analytic function of $z$. The following theorem proves this and slightly more: instead of continuity jointly in $t$ and $z$ it is enough to require boundedness.

ThEOREM 4. A function $x(t, z)$ defines a function $x(\cdot, z)$ regular in a region $D$ if and only if

(a) $x(t, z)$ is continuous on $[a, b]$ for each $z$ in $D$;

\footnotetext{
${ }^{2}$ Compact sets, as we use the term, are closed.
} 
(b) $x(t, z)$ is regular in $D$ for each $t$ on $[a, b]$;

(c) to each compact set $S$ in $D$ there corresponds a constant $M$ such that $|x(t, z)| \leqq M$ if $t$ is on $[a, b]$ and $z$ is in $S$.

By employing Cauchy's integral formula much as in the proof of Theorem 1 we can show directly that the difference quotient

$$
(x(t, z+h)-x(t, z)) / h
$$

converges to its limit as $h \rightarrow 0$, uniformly with respect to $t$. But this means that

$$
(x(\cdot, z+h)-x(\cdot, z)) / h
$$

converges to a limit in the topology of $C$. The conditions (a)-(c) are thus seen to be sufficient. Their necessity is trivial.

The continuity of $x(t, z)$ and $\partial x / \partial z$ jointly in $t$ and $z$ is a consequence of the regularity of $x(\cdot, z)$.

The situation in the space $L^{p}$ is much more complicated. Wiener [1] studied power series in $z$ whose coefficients are functions in $L^{2}$. Taylor [6, Theorem 3.2] showed that a function $F(z)$ which is analytic in $D$, with values in $L^{p}$, can be represented by a function $x(t, z)$ which is defined when $z$ is in $D$ and $t$ is on the interval $[a, b]$, but not in a certain fixed set $N$ of measure zero. The significant thing is that this exceptional set is independent of $z$. The function $x(t, z)$ is regular as a function of $z$, and the integral $\int_{a}^{b}|x(t, z)|^{p} d t$ is bounded in each compact subset of $D$. The latter conditions are, conversely, sufficient to determine $x(\cdot, z)$ as an analytic function on $D$ in to $L^{p}$.

In the paper referred to above, Taylor studied abstract linear differential systems of the forms

$$
d x / d \lambda=A_{\lambda} x \text { and } d x / d \lambda=A_{\lambda} x+z(\lambda),
$$

where $A_{\lambda}$ is a linear transformation depending analytically on $\lambda$, and $z(\lambda)$ is a given abstract function of $\lambda$. In concrete realizations of the abstract theory such systems include a variety of types of equations, such as integro-differential equations, differential equations in an infinity of unknowns, and so forth.

5. Singularities. Isolated singularities may be classified, as in classical theory, under the headings: (1) removable singularities, (2) poles, (3) essential singularities. An isolated singularity is removable if it disappears when a proper definition is given to the function at the point in question. We can prove Riemann's theorem: an isolated singularity $z_{0}$ is removable if $\|x(z)\|$ is bounded in the neighborhood of $z_{0}$. An isolated singularity $z_{0}$ is a pole if, for a suitable positive 
integer $m,\left(z-z_{0}\right)^{m} x(z)$ has a removable singularity at $z_{0}$. The smallest admissible integer $m$ is called the order of the pole. An equivalent definition may be based upon the nature of the Laurent expansion about the point $z_{0}$. By Lemma 1 and Riemann's theorem we may show that if, for each $f$ in $E^{*}, f x(z)$ has a pole of order not greater than $m$ at $z_{0}$, then $x(z)$ has a pole of order not greater than $m$; and if the pole is of order exactly $m$, then there is at least one $f$ in $E^{*}$ such that $f x(z)$ has a pole of order $m$ at $z_{0}$.

Let us consider spaces with denumerable bases. It appears from Theorem 3 that in such a space the singularity of a function $x(z)$ may arise either from a singularity of one of the component functions $u_{n}(z)$ or from the behavior of the series

$$
x(z)=\sum_{k=1}^{\infty} u_{k}(z) x_{k}
$$

as a whole. As an example, consider the Hilbert space $l^{2}$, and the function $x(z)$ with components $u_{n}(z)=z^{n}$. Since

$$
\|x(z)\|^{2}=\sum_{k=1}^{\infty}|z|^{2 k},
$$

it is clear that $x(z)$ is regular if $|z|<1$, but not defined if $|z| \geqq 1$. The component functions, individually, give no indication of the fact that $|z|=1$ is a natural boundary.

We can prove, however, that an isolated singularity is always traceable to one of the components.

THEOREM 5. Suppose that the conditions of Theorem 3 obtain in a region $D: 0<\left|z-z_{0}\right|<R$, and suppose that each of the functions $u_{n}(z)$ is, in addition, regular at $z_{0}$. Then the series $\sum_{k=1}^{\infty} u_{k}(z) x_{k}$ converges if $\left|z-z_{0}\right|<R$, and defines a function regular, not only in $D$, but at $z_{0}$.

To prove the theorem, consider an element $f$ of $E^{*}$, and the series

$$
f x(z)=\sum_{k=1}^{\infty} u_{k}(z) f\left(x_{k}\right) .
$$

The terms of the series are regular if $0 \leqq\left|z-z_{0}\right|<R$. If $0<R_{1}<R$, there exists a constant $M$ such that

$$
\left|\sum_{k=1}^{n} u_{k}(z) f\left(x_{k}\right)\right| \leqq M
$$

if $\left|z-z_{0}\right|=R_{1}$ and $n=1,2, \cdots$. The same inequality must hold if 
$0 \leqq\left|z-z_{0}\right|<R_{1}$, by the maximum modulus theorem. Hence, letting $n \rightarrow \infty$, we deduce that $|f x(z)| \leqq M$ if $0<\left|z-z_{0}\right| \leqq R_{1}$. By Lemma 1 we know that $x(z)$ is bounded in the same range of $z$. By Riemann's theorem, then, the possible singularity at $z_{0}$ is removable. The conclusion of the theorem is now easily proved.

6. Resolvents. It has long been recognized that the methods of the theory of functions of a complex variable are important in the theory of linear transformations. F. Riesz, in his monograph on the subject of infinite systems of linear equations (F. Riesz [1, pp. 117-121]) indicates briefly the potentialities of the calculus of residues as a means of studying the spectrum and the characteristic manifolds of a linear transformation.

Let $T$ be a linear transformation of the Banach space $E$ into itself. If the transformation $T_{\lambda} \equiv T-\lambda I$ ( $I$ the identity) maps $E$ into itself in a one-to-one fashion, the inverse transformation, denoted by $R_{\lambda}$, will also be linear. We shall call $R_{\lambda}$ the resolvent of $T$, and the set of values of $\lambda$ for which $R_{\lambda}$ is defined the resolvent set $R$. All values of $\lambda$ not in $R$ belong to the spectrum of $T$. A number $\lambda$ is said to be in the point spectrum of $T$ if it is a characteristic value, that is, if there is an $x \neq 0$ such that $T x=\lambda x$.

Our discussion will center around $R_{\lambda}$ as a function of $\lambda$. As far as questions of analyticity are concerned, it is immaterial whether we study $R_{\lambda}$ or $R_{\lambda} x$, because of Theorem 2 . We shall study $R_{\lambda}$ directly.

THEOREM 6. The resolvent $R_{\lambda}$ is analytic and regular in the resolvent set (which is open, but not necessarily connected). If $\lambda$ and $\mu$ are in $R$,

$$
\begin{aligned}
R_{\lambda}-R_{\mu} & =(\lambda-\mu) R_{\lambda} R_{\mu}, \\
\left(d^{n} / d \lambda^{n}\right) R_{\lambda} & =n ! R_{\lambda}^{n+1} .
\end{aligned}
$$

The set $R$ contains every $\lambda$ such that $|\lambda| \geqq\|T\|$, and for these values

$$
R_{\lambda}=-\sum_{n=1}^{\infty} \lambda^{-n} T^{n-1}
$$

If $C$ is a contour enclosing the spectrum of $T$, then

$$
T=-\frac{1}{2 \pi i} \int_{C} \lambda R_{\lambda} d \lambda .
$$

Formula (1) is the well known functional equation of the resolvent, familiar in the theory of integral equations. Formula (3) is the Neumann expansion of the resolvent, in abstract form. Formula (4) ap- 
pears to have been overlooked until now. For the proof of (1)-(3), see Taylor [2]. To obtain (4), multiply (3) by $\lambda$ and integrate, after deforming the contour, if necessary. Other references are: Stone [1, pp. 139-141]; Hildebrandt [1, pp. 195-199]; Nagumo [1]; Taylor [6, pp. 590-593]; Lorch [1].

The resolvent set is the maximal domain of regularity of $R_{\lambda}$. All the singular points of $R_{\lambda}$ belong to the spectrum of $T$, and a point of the spectrum is a singularity of $R_{\lambda}$, provided that it is a limit point of the resolvent set. The resolvent $R_{\lambda}$ is regular at infinity, and vanishes there, by (3). Hence, by Liouville's theorem, it is either identically zero (in the trivial case that the space $E$ consists of a single element), or the spectrum of $T$ contains at least one point.

With each simple closed contour $C$ lying in $R$ we may associate a transformation $P$ defined as follows:

$$
P=-\frac{1}{2 \pi i} \int_{C} R_{\lambda} d \lambda
$$

These transformations play a fundamental rôle in the study of the singularities of $R_{\lambda}$. Their use in studying the spectrum has recently been indicated by Lorch. We summarize the salient properties of these transformations in a theorem (Lorch [1, Theorems 5 and 6]).

Theorem 7. The transformation $P$ is a projection, that is, $P^{2}=P$. It is permutable with $T$. If $C_{1}$ and $C_{2}$ are two contours in $R$, and if $P_{1}, P_{2}$ are the corresponding projections, $P_{1} P_{2}=P_{2} P_{1}$, and $P_{1} P_{2}=P_{1}$ if $C_{1}$ lies inside $C_{2}$, while $P_{1} P_{2}=0$ if $C_{1}$ lies outside $C_{2}$.

In proving this theorem, and in many other calculations involving contour integrals, equation (1) provides a valuable key. It may be shown that $P=I$ if and only if the spectrum of $T$ lies within $C$, and that $P=0$ if and only if the spectrum of $T$ lies outside $C$.

A partial analysis of the structure of $T$ and $R_{\lambda}$ is possible with the aid of $P$. There is not space in this paper for a detailed account. Let us consider merely the case of an isolated point $\lambda_{0}$ of the spectrum of $T$, that is to say, an isolated singularity of $R_{\lambda}$. Let $C$ be a small contour inclosing $\lambda_{0}$ but no other point of the spectrum. Then the Laurent expansion of $R_{\lambda}$ is

$$
R_{\lambda}=\sum_{n=0}^{\infty}\left(\lambda-\lambda_{0}\right)^{n} A_{n}+\sum_{n=1}^{\infty}\left(\lambda-\lambda_{0}\right)^{-n} B_{n},
$$

where 


$$
B_{n}=\frac{1}{2 \pi i} \int_{C}\left(\lambda-\lambda_{0}\right)^{n-1} R_{\lambda} d \lambda, \quad n=0, \pm 1, \pm 2, \cdots
$$

and we understand that $A_{n}=B_{-n}$. One can show that $B_{n+1}=T_{\lambda_{0}} B_{n}$, $n=1,2,3, \cdots$. Also, $B_{1}=-P$, and so

$$
B_{n}=-T_{\lambda_{0}}^{n-1} P, \quad n=1,2,3, \cdots \text {. }
$$

The simplest case is that in which $\lambda_{0}$ is a pole of $R_{\lambda}$.

THEOREM 8. If $\lambda_{0}$ is a pole of $R_{\lambda}$, it is in the point spectrum of $T$. If $x$ is a corresponding characteristic element, $P x=x$. A sufficient, but not necessary, condition for $\lambda_{0}$ to be a pole is that $P$ project $E$ into a finite dimensional linear manifold.

To prove the first statement, let $m$ be the order of the pole. Then from (6) we infer that $B_{m} \neq 0$ and $B_{n}=0$ if $n>m$. Let $y$ be chosen so that $x=B_{m} y \neq 0$. Then $T_{\lambda_{0}} x=B_{m+1} y=0$, by (7). We may also prove that $A_{0} T_{\lambda_{0}}=I-P$. Hence we see that $P x=x$.

To prove the second part of the theorem, we define

$$
S_{\lambda}=\sum_{n=1}^{\infty}\left(\lambda-\lambda_{0}\right)^{-n} B_{n} .
$$

It is easily proved that $S_{\lambda}$ is regular if $\lambda \neq \lambda_{0}$, and that $S_{\lambda} P=S_{\lambda}$. Furthermore,

$$
S_{\lambda} T_{\lambda}=P .
$$

Now suppose that the range $E_{0}$ of $P$ is finite dimensional. Within $E_{0}$, $P$ coincides with the identity. From the above remarks we therefore conclude that $S_{\lambda}$ is essentially a transformation of $E_{0}$ into itself, and that it is, within $E_{0}$, the resolvent of $T$. Its singularity at $\lambda_{0}$ is therefore that of the resolvent of a finite matrix, namely, a pole.

There is a large class of transformations $T$ for which the only singularities of $R_{\lambda}$, aside from $\lambda=0$, are poles. The most familiar case is that when $T$ is completely continuous. It is easy, in this case, to show that the only possible limit point of the spectrum is the origin. For an isolated singularity $\lambda_{0} \neq 0$ it is readily proved that $P$ is completely continuous and that its range $E_{0}$ is finite dimensional. Theorem 8 then shows that $\lambda_{0}$ is a pole of the resolvent. This argument has been pointed out by several people (Schauder [1, p. 193]; Hildebrandt [1, p. 198]; Nagumo [1, pp. 79-80]). An extension to transformations which are in a sense "nearly" completely continuous has been indicated in various places (Hildebrandt, loc. cit.; Nikolskij [1]; Yoshida $[1,2])$. 
In case the only singularities of $R_{\lambda}$ aside from $\lambda=0$ are poles, the transformation $z=\lambda^{-1}$ carries $R_{\lambda}$ into a meromorphic function which may be expanded by Mittag-Leffler's theorem. If the number of poles is finite, $R_{\lambda}$ is rational, and we get an explicit partial fraction representation. In fact, since $R_{\lambda}$ vanishes at infinity, it is equal to the sum of its principal parts at the poles $\lambda_{1}, \cdots, \lambda_{n}$. If we enclose each pole in a small contour $C_{j}$, and denote the corresponding projection (5) by $P_{j}$, we find, from (6), that

$$
R_{\lambda}=-\sum_{j=1}^{n} \sum_{k=1}^{m_{j}}\left(\lambda-\lambda_{j}\right)^{-k} T_{\lambda_{j}}^{k-1} P_{j},
$$

where $m_{j}$ is the order of the pole $\lambda_{j}$. By integrating around a contour enclosing all the poles we find the relation

$$
P_{1}+\cdots+P_{n}=I \text {. }
$$

Finally, by combining (4) and (10), we find that if $\lambda_{1}, \cdots, \lambda_{r}$ are simple poles, while $\lambda_{r+1}, \cdots, \lambda_{n}$ are multiple poles,

$$
T=\sum_{j=1}^{r} \lambda_{j} P_{j}+T \sum_{j=r+1}^{n} P_{j} .
$$

Formulas (10)-(12) generalize certain results in the theory of finite matrices (Frobenius [1]; Schwerdtfeger [1, 2, 3]). In case the poles are all simple, $T$ is completely reducible. This is the abstract generalization of the reduction of a matrix to diagonal form.

It is of some interest to record that if $E$ is the Hilbert space $l^{2}$, and if the resolvent is defined by an infinite matrix with elements $R_{i j}(\lambda)$, then if $T$ is either Hermitian or unitary, the spectrum of $T$ consists of the singular points of the functions $R_{i j}(\lambda)$, together with the limit points of such singularities. This does not hold true for an arbitrary $T$, however (Wintner [1, pp. 178 and 213-214]).

7. Functions of linear transformations. Formula (4) of $\$ 6$ may be generalized as follows: Let $D$ be a fixed simply connected open set containing the spectrum of $T$. Let $\mathcal{F}$ be the class of all single-valued numerical functions $F(\lambda)$ which are holomorphic in $D$. This class $\mathcal{f}$ is a commutative ring. Let us define

$$
F(T)=-\frac{1}{2 \pi i} \int_{C} F(\lambda) R_{\lambda} d \lambda,
$$

where $C$ is a contour lying in $D$ and surrounding the spectrum of $T$. 
If $F(\lambda)=\sum_{n=0}^{\infty} a_{n} \lambda^{n}$ is an entire function, formula (3) of $\S 6$ enables us to show that $F(T)=\sum_{n=0}^{\infty} a_{n} T^{n}$.

THEOREM 9. The class of all linear transformations $F(T)$ generated by (1) is a commutative sub-ring of the ring of all linear transformations of $E$ into itself. The mapping defined by (1) is a homomorphism.

The proof of the theorem consists in showing that the correspondence $F(\lambda) \rightarrow F(T)$ preserves sums and products. The preservation of sums is trivial. Suppose that $F(\lambda)$ and $G(\lambda)$ are in $\mathcal{F}$. If $\Gamma$ is a contour in $D$, enclosing $C$, and $\lambda$ is on $C$,

$$
G(\lambda)=\frac{1}{2 \pi i} \int_{\Gamma} \frac{G(\mu)}{\mu-\lambda} d \mu .
$$

If we substitute the above formula into the integral

$$
-\frac{1}{2 \pi i} \int_{C} F(\lambda) G(\lambda) R_{\lambda} d \lambda
$$

reverse the order of integration, and use formula (1) of $\S 6$, the above integral around $C$ is found to have the value $F(T) G(T)$. This proves that products are preserved by (1).

An immediate and important consequence of Theorem 9 is that if $F(\lambda)$ belongs to $\mathcal{F}$ and has no zeros in $D$, then $F(T)$ has a multiplicative inverse $[F(T)]^{-1}$; the inverse corresponds to $1 / F(\lambda)$, and may be written $I / F(T)$.

Formula (1) may be written in the equivalent form

$$
F(T)=\frac{1}{2 \pi i} \int_{C} \frac{F(\lambda)}{\lambda I-T} d \lambda,
$$

by recalling that $R_{\lambda}=(T-\lambda I)^{-1}=-(\lambda I-T)^{-1}$. In the finite dimensional case this formula seems to be due to Poincaré, who used it in the study of continuous groups (Poincaré $[1,2,3])$. It has appeared elsewhere (Buhl [1, pp. 37-38]; Schwerdtfeger [1, p. 311]; Giorgi $[1$, p. 7$]$; Wedderburn $[1$, p. 130]). A special case of the formula was used by Hille [1, pp. 3 and 22] in studying semi-groups of transformations in Banach spaces. Theorem 9 seems to be new, and apparently formula (1) itself is also new in the generality with which it is presented here.

As an interesting application, we shall use the above theory to solve the linear differential equation

$$
d^{n} x / d t^{n}+a_{1} d^{n-1} x / d t^{n-1}+\cdots+a_{n} x=y(t)
$$


with the initial conditions

$$
x(0)=x^{\prime}(0)=\cdots=x^{(n-1)}(0)=0 .
$$

The coefficients $a_{1}, \cdots, a_{n}$ are assumed to be constants, and $y(t)$ is a given function, continuous on the interval $[0,1]$.

If $C$ is the Banach space of functions $x(t)$ continuous on $[0,1]$, let $T x(\cdot)=y(\cdot)$ mean that

$$
y(t)=\int_{0}^{t} x(s) d s .
$$

One may show without trouble that the solution of the integral equation

is

$$
x(t)-\lambda \int_{0}^{t} x(s) d s=y(t)
$$

$$
x(t)=-\frac{1}{\lambda} y(t)-\frac{1}{\lambda^{2}} \int_{0}^{t} e^{(t-s) / \lambda} y(s) d s .
$$

The spectrum of $T$ consists of the single point $\lambda=0$, and the resolvent of $T$ is defined by (6).

The differential system (3) and (4) is equivalent to the equation

$$
x+a_{1} T x+\cdots+a_{n} T^{n} x=T^{n} y .
$$

If $F(\lambda)=1+a_{1} \lambda+\cdots+a_{n} \lambda^{n}$, (7) becomes $F(T) x=T^{n} y$. Since $F(\lambda)$ and $1 / F(\lambda)$ are regular at $\lambda=0$, and hence in the neighborhood of the spectrum of $T$, the inverse of $F(T)$ exists, and the solution of $(7)$ is

$$
x=\left(T^{n} / F(T)\right) y .
$$

Thus, using formula (1),

$$
x=-\frac{1}{2 \pi i} \int_{C} \frac{\lambda^{n}}{F(\lambda)} R_{\lambda} y d \lambda,
$$

where $C$ is a contour enclosing $\lambda=0$ and excluding the zeros of $F(\lambda)$. Since the resolvent is defined by (6), (8) gives us an explicit means of calculating $x(t)$. It is convenient to make the substitution $z=\lambda^{-1}$. We then obtain

$$
x(t)=\frac{1}{2 \pi i} \int_{0}^{t} y(s) d s \int_{\Gamma} \frac{e^{z(t-s)}}{z^{n}+a_{1} z^{n-1}+\cdots+a_{n}} d z,
$$

where $\Gamma$ is a contour enclosing the roots of the polynomial $z^{n}+a_{1} z^{n-1}$ 
$+\cdots+a_{n}$. Formula (9) is due originally to Cauchy (see Darboux [1]).

8. Analytic functions of an abstract variable. In this section we shall survey briefly the theory of analytic functions where both the domain and range of the functions are Banach spaces. This theory has its roots in ideas which go back to Volterra and other pioneers in functional analysis. The most important early impetus was given by Fréchet, who in a series of papers developed the notions of functional polynomials and power series $[1,2,4]$ and pointed the way to a successful calculus based upon his definition of a differential [3]. The work of Gateaux $[1,2]$ sketched the outlines of a theory of analytic functionals. The first systematic abstract theory of analytic functions was developed by Martin [1]. He was a pupil of A. D. Michal, under whose guidance an intensive study of the whole field of analysis and geometry in abstract spaces has been carried on for more than a decade (see the interpretation of the abstract point of view in Michal [2], and the references to the literature in Michal [1]). Various applications of the theory, in the shape given it by Martin, have appeared (Michal and Clifford [1]; Michal and Martin [1]).

An abstract theory of analytic functions, along the lines indicated by Gateaux, was announced by Graves [1], and independently by Taylor ([1 and 5]; see especially the footnote on p. 466 of [5]). In Martin's theory the basic notion is that of a power series expansion. The terms of a power series are homogeneous polynomials. For the literature on polynomials, see Fréchet [1, 4]; Martin [1]; Mazur and Orlicz [1]; Highberg [1, 2]; Taylor [3]; Banach [2]. In the theory of Graves and Taylor, $F(x)$ is said to be holomorphic in a region $D$ if it is continuous there and if the limit

$$
\delta F(x ; y)=\lim _{\lambda \rightarrow 0}(F(x+\lambda y)-F(x)) / \lambda
$$

exists for each $y$ in the Banach space and each $x$ in the region $D$. It can be shown that $\delta F(x ; y)$ is the Fréchet differential, and that $F(x)$ is analytic in the sense of Martin. The two different approaches result in the same general theory. There are certain connections between the above ideas and the work of Fantappié $[1,2]$ on analytic functionals. Wintner's theory of regular power series [2] is closely allied to the abstract theory described above.

One illustration of the general theory is to be found in the study of resolvents. If we regard the linear transformation $T$ as a variable, and denote the resolvent by $R(\lambda, T)$, the resolvent is an analytic 
function, not only of $\lambda$, but of $T$ as well. ${ }^{3}$ The Fredholm theory provides an explicit exhibition of the dependence of the resolvent upon $\lambda$ and $T$. An abstract treatment of the Fredholm determinant and first minor was given by Michal and Martin [1]. Closely allied results, for transformations of finite norm in Hilbert space, were given by Smithies [1], though without any explicit use of a theory of abstract analytic functions. It would seem to be worth while to pursue the study of $R(\lambda, T)$ and of the functions $F(T)$ defined in $\$ 7$ from this general point of view.

A theory of analytic functions from Riemann's point of view may be built up by starting from a real Banach space $B$, and constructing a complex Banach space in much the same way that the complex numbers are formed from the real numbers. The only part of the construction which offers the least difficulty is the defining of a suitable norm of the complex element $x+i y$, where $x$ and $y$ are in the real space $B$. That $\left(\|x\|^{2}+\|y\|^{2}\right)^{1 / 2}=\|x+i y\|$ will not do, in general, was pointed out in a paper by Michal, Davis, and Wyman [1]. A satisfactory definition is the following, due to Taylor (Michal and Wyman $[1$, p. 249]):

$$
\|x+i y\|=\sup _{\|f\|=1}\left(|f(x)|^{2}+|f(y)|^{2}\right)^{1 / 2}, \quad f \in B^{*} .
$$

With the complex Banach space so constructed it is possible to develop analogues of the Cauchy-Riemann equations, and a corresponding notion of biharmonic functions (Taylor [4]). It is also possible to extend a "real" analytic function, defined by a series of homogeneous polynomials, into the complex Banach space (Taylor [3, pp. 312-315]). This process utilizes certain relations between abstract polynomials and their polars. There are various unsolved problems connected with bounds and inequalities which make the present state of this work unsatisfactory.

There is a converse problem: can every complex Banach space $E$ be decomposed into a real and an.imaginary part? The question may be put in the following form (cf. Michal and Wyman [1, Theorem 3]): does there exist a continuous transformation $T$ of $E$ into itself, having the properties $T^{2} x=x, T(x+y)=T x+T y, T(a x)=\bar{a} T x$ ? We may call such a transformation a conjugation. Let us call a subset $M$ of $E$ a maximal essentially real linear manifold if

\footnotetext{
${ }^{3}$ In this connection we note that a function $F(\lambda, x)$ of a complex variable $\lambda$ and a Banach variable $x$ is analytic in the pair $\lambda, x$ if it is analytic in each variable separately (Taylor [5, Theorem 3.2]). Whether the like is true of functions of two abstract variables is at present unknown.
} 
(a) it is closed under addition and under multiplication by real numbers;

(b) $x$ and $i x$ are both in $M$ only if $x=0$;

(c) $M$ is not a proper subset of any set with properties (a) and (b). Then it is not difficult to show that there exists a conjugation of $E$ if and only if there exists in $E$ a closed and maximal essentially real linear manifold. This whole problem has been studied by Taylor [7], but no complete solution has been reached.

\section{BIBLIOGRAPHY}

S. BANACH

1. Opérations linéaires, Warsaw, 1932.

2. Über homogene polynome in $\left(L^{2}\right)$, Studia Mathematica vol. 7 (1938) pp. 36-44. M. BôCHER

1. On semi-analytic functions of two variables, Ann. of Math. vol. 12 (1911) pp. 1826.

H. F. Bohnenblust AND A. Sobczyk

1. Extensions of functionals on complex linear spaces, Bull. Amer. Math. Soc. vol. 44 (1938) pp. 91-93.

A. BuHL

1. Gravifiques, groupes, mécaniques, Mémorial des Sciences Mathématiques vol. 62 (1934).

G. DARBoUX

1. Application de la méthode prêcédente à l'équation linéaire d coefficients constants avec second membre, Bull. Sci. Math. vol. 3 (1879) pp. 325-328.

N. DUNFORD

1. Uniformity in linear spaces, Trans. Amer. Math. Soc. vol. 44 (1938) pp. 305-356.

N. Dunford ANd A. P. Morse

1. Remarks on the preceding paper of James A. Clarkson, Trans. Amer. Math. Soc. vol. 40 (1936) pp. 415-420.

L. FantaPpie

1. I funzionali analitici, Memorie della Reale Accademia Nazionale dei Lincei vol. 3 no. 11 (1930).

2. Überblick über die Theorie der analytischen Funktionale und ihre Anwendungen, Jber. Deutschen Math. Verein. vol. 43 (1933) pp. 1-25.

M. FRÉCHET

1. Une défnition fonctionelle des polynomes, Nouvelles Annales de Mathématiques vol. 9 (1909) pp. 145-162.

2. Sur les fonctionelles continues, Ann. École Norm. vol. 27 (1910) pp. 193-216.

3. La notion de différentielle dans l'analyse générale, Ann. École Norm. vol. 42 (1925) pp. 293-323.

4. Les polynomes abstraits, J. Math. Pures Appl. vol. 8 (1929) pp. 71-92.

G. Frobenius

1. Über vertauschbare Matrizen, Berlin Math. Ges. Sitzungsber., 1896, pp. 601-614.

R. GateauX

1. Fonctions d'une infinité de variables indépendantes, Bull. Soc. Math. France vol. 47 (1919) pp. 70-96. 
2. Sur les fonctionelles continues et les fonctionelles analytiques, Bull. Soc. Math. France vol. 50 (1922) pp. 1-21.

G. GIORGI

1. Nuove osservazioni sulle funzioni delle matrici, Rendiconti della Reale Accademia Nazionale dei Lincei (6) vol. 8 (1928) pp. 3-8.

L. M. Graves

1. Topics in the functional calculus, Bull. Amer. Math. Soc. vol. 41 (1935) pp. 641662.

I. E. Highberg

1. Polynomials in abstract spaces, California Institute of Technology thesis, 1936.

2. A note on abstract polynomials in complex spaces, J. Math. Pures Appl. vol. 16 (1937) pp. 307-314.

T. H. HILDEBRANDT

1. Linear functional transformations in general spaces, Bull. Amer. Math. Soc. vol. 37 (1931) pp. 185-212.

E. HILle

1. Notes on linear transformations. II. Analyticity of semi-groups, Ann. of Math. vol. 40 (1939) pp. 1-47.

E. R. LORCH

1. The spectrum of linear transformations, Trans. Amer. Math. Soc. vol. 52 (1942) pp. 238-248.

R. S. MARTIN

1. Contributions to the theory of functionals, California Institute of Technology thesis, 1932.

S. MazUr and W. ORLICZ

1. Grundlegende Eigenschaften der polynomische operationen, Studia Mathematica vol. 5 (1935) pp. 50-68 and 179-189.

A. D. Michal

1. General differential geometries and related topics, Bull. Amer. Math. Soc. vol. 45 (1939) pp. 529-563.

2. Recent general trends in mathematics, Science vol. 92 (1940) pp. 563-566.

A. D. Michal and A. H. Clifford

1. Fonctions analytiques implicites dans des espaces vectoriels abstraits, C. R. Acad. Sci. Paris vol. 197 (1933) pp. 735-737.

A. D. Michal, R. Davis, M. Wyman

1. Polygenic functions in general analysis, Annali della R. Scuola Normale di Pisa vol. 9 (1940) pp. 97-108.

A. D. Michal and R. S. Martin

1. Some expansions in vector space, J. Math. Pures Appl. vol. 13 (1934) pp. 69-91.

A. D. Michal and Max Wyman

1. Characterization of complex couple spaces, Ann. of Math. vol. 42 (1941) pp. 247250.

M. NAGUMo

1. Einige analytische untersuchungen in linearen metrischen Ringen, Jap. J. Math. vol. 13 (1936) pp. 61-80.

S. Nikolskij

1. C. R. (Doklady) Acad. Sci. URSS. vol. 16 (1926) pp. 315-319.

H. Poincare

1. Sur les groupes continus, Transactions of the Cambridge Philosophical Society 
vol. 18 (1899) pp. 220-255; reprinted in the collected works, vol. 3, pp. 173-212, Paris, 1934.

2. Quelques remarques sur les groupes continus, Rend. Circ. Mat. Palermo vol. 15 (1901) pp. 321-368; reprinted in the collected works, vol. 3, pp. 213-260, Paris, 1934.

3. Nouvelles remarques sur les groupes continus, Rend. Circ. Mat. Palermo vol. 25 F. RIESZ (1908) pp. 81-130; reprinted in the collected works, vol. 3, pp. 261-321.

1. Les systèmes d'équations linéaires à une infinité d'inconnus, Paris, 1913.

2. Über lineare Funktionalgleichungen, Acta Math. vol. 41 (1918) pp. 71-98.

J. SchaUdER

1. Über lineare vollstetige Funktionaloperationen, Studia Mathematica vol. 2 (1930) pp. 183-196.

H. SCHWERDTFEGER

1. Sur une formule de $H$. Poincarê relative a la theorie des groupes de S. Lie, Enseignement Mathématique vol. 32 (1933) pp. 304-319.

2. Beiträge zum Matricen-Kalcül und zur Theorie der Gruppenmatrix, InauguralDissertation, Bonn, 1935, pp. 1-51.

3. Über mehrdeutige Matrixfunktionen, Compositio Math. vol. 3 (1936) pp. 380390.

F. SMithies

1. The Fredholm theory of integral equations, Duke Math. J. vol. 8 (1941) pp. 107130.

M. H. STONE

1. Linear transformations in Hilbert space, Amer. Math. Soc. Colloquium Publications vol. 15, New York, 1932.

J. D. TAMARKIN

1. On Fredholm's integral equations whose kernels are analytic in a parameter, Ann. of Math. vol. 28 (1926) pp. 127-152.

A. E. TAYLOR

1. Analytic functions in general analysis, Annali della R. Scuola Normale Superiore di Pisa vol. 6 (1937) pp. 277-292.

2. The resolvent of a closed transformation, Bull. Amer. Math. Soc. vol. 44 (1938) pp. 70-74.

3. Additions to the theory of polynomials in normed linear spaces, Tôhoku Math. J. vol. 44 (1938) pp. 302-318.

4. Biharmonic functions in abstract spaces, Amer. J. Math. vol. 60 (1938) pp. 416422.

5. On the properties of analytic functions in abstract spaces, Math. Ann. vol. 115 (1938) pp. 466-484.

6. Linear operations which depend analytically on a parameter, Ann. of Math. vol. 39 (1938) pp. 574-593.

7. Conjugations of complex linear spaces, University of California Publications in Mathematics (in press).

J. H. M. WEDDERBURN

1. Lectures on matrices, Amer. Math. Soc. Colloquium Publications vol. 17, New York, 1934.

N. WIENER

1. Note on a paper of M. Banach, Fund. Math. vol. 4 (1923) pp. 136-143. 
A. WINTNER

1. Spectraltheorie der unendlichen Matrizen, Leipzig, 1929.

2. Upon a theory of infinite systems of nonlinear implicit and differential equations, Amer. J. Math. vol. 53 (1931) pp. 241-257.

K. YoshidA

1. Abstract integral equations and the homogeneous stochastic process, Proc. Imp. Acad. Tokyo vol. 14 (1938) pp. 286-291.

2. Quasi-completely continuous linear functional operations, Jap. J. Math. vol. 15 (1939) pp. 297-301.

UNIVERSity OF CALIFORNIA, Los ANgeles, Calif. 